\title{
LAS TECNOLOGÍAS DE INFORMACIÓN Y COMUNICACIÓN (TIC) COMO RESPUESTA A NECESIDADES EDUCATIVAS DEL MEDIO RURAL
}

\author{
Marlene Víquez Salazarl \\ Docente de la Universidad Estatal a Distancia (UNED) \\ San José, Costa Rica
}

Recibido: 15 de junio 2007 • Aprobado: 20 de agosto 2007

\begin{abstract}
Resumen: El artículo tiene como propósito reflexionar, desde una perspectiva crítica, sobre el uso de las tecnologías de la información y las comunicaciones (TIC) en la educación rural, bajo el supuesto de que estos recursos no resuelven por sí solos ls problemas, sino que pueden generar respuestas a situaciones concretas en el ámbito de la educación rural. Para ello, se hace un análisis desde diferentes aristas.
\end{abstract}

Palabras clave: Necesidades educativas, zonas rurales costarricenses, TIC en procesos educativos, potencial de TIC para educación rural.

Abstract: The article must like intention reflect, from a critical perspective, on the use of the technologies of the information and the communications (TIC) in the rural education, under the assumption that these resources are not in themselves solutions to problems, but that can allow to obtain solutions to concrete problems, in the scope of the rural education. For it, an analysis becomes from different edges.

Key words: Educative needs, costarrican rural zones, TIC in education proceses, TIC potencial in the rural education.

\section{INTRODUCCIÓN}

Es un hecho que las nuevas tecnologías de la información y la comunicación (TIC), así como la sociedad informatizada o del conocimiento, que se impulsa desde finales del siglo pasado, está generando, con una velocidad sorprendente, una transformación en las relaciones culturales entre las personas: cambios en las formas de actuar, pensar, sentir, comunicarse y aprender.

\footnotetext{
Educadora, graduada en la Universidad de Costa Rica (UCR). Máster en Educación Rural Centroamericana, Universidad Nacional (UNA). Ha trabajado como asesora en proyectos en telecomunicaciones, en la formación y capacitación de educadores y, como autora y editora de textos educativos. Actualmente es profesora de la Universidad Estatal a Distancia (UNED). Correo electrónico: mviquez@uned.ac.cr mavisa1951@hotmail.com
} 
Como consecuencia de ello, en el campo educativo emerge una serie de inquietudes relacionadas con el uso de estos nuevos recursos en los procesos escolares. En el debate existe una variedad de opiniones.

Por una parte, se hace un llamado a valorar con cautela los efectos de dichos recursos en los procesos educativos en general. Se afirma que si este impacto tecnológico no se enfrenta de manera adecuada, “...puede llevar a una pérdida del sentido de la realidad...” (UNESCO, 1996, p. 69); pero el no hacerlo, puede también incrementar las desigualdades entre las poblaciones que tienen acceso a estas herramientas y las que no. Preocupa, además, como el uso continuo de estos recursos en los salones escolares podría afectar los procesos de socialización entre los niños, entre las niñas, entre los jóvenes y entre los estudiantes y el docente.

Por lo tanto, se les sugiere, a los países en vía de desarrollo, un replanteamiento de sus sistemas educativos nacionales, de sus programas y de lo que acontece en los procesos educativos escolares, con la finalidad de que la formación que estén recibiendo los niños, las niñas y los jóvenes, responda a este nuevo entorno mundial. En este sentido, se impulsa una educación que les proporcione a los estudiantes "...los medios de dominar la proliferación de las informaciones, esto es, de seleccionarlas y jerarquizarlas dando muestras de sentido crítico...” (UNESCO, 1996, p. 70).

En general, la literatura a favor del uso de las TIC en la educación, considera que estos recursos incrementan el logro de los objetivos de aprendizaje por los cambios que promueve en la mediación pedagógica, por la diversidad de experiencias novedosas que ofrecen, por la posibilidad que brinda para que el estudiantado avance de acuerdo con sus necesidades e intereses. Se argumenta, además, que dichos recursos facilitan la educación permanente, lo cual es clave para mantenerse en el contexto laboral.

Desde otra perspectiva, el autor afirma que estas “...promesas de las TIC en educación están lejos de ser realidad...” (Tedesco, 2003, p. 10). Justifica la incorporación de tales recursos en las políticas educativas democráticas pero teniendo presente que el ejercicio de la potencialidad democratizadora o innovadora que se les asigna "...no depende de las tecnologías mismas sino de los modelos sociales y pedagógicos en los cuales se las utilice” (Tedesco, 2003, p. 10). El mismo autor agrega que el no hacerlo, “...puede condenar a la marginalidad a todos los que queden fuera del dominio de los códigos que permitan manejar estos instrumentos...” (Tedesco, 2003 p. 10).

Ante el contexto descrito, la finalidad de este artículo consiste en contribuir al debate sobre el papel que podrían jugar las TIC en respuesta a las necesidades educativas en el medio rural costarricense, y de esta forma, ampliar las posibilidades de desarrollo personal de estudiantes, educadores y comunidades.

\section{LA COMPUTADORA Y LA INTERNET EN LA EDUCACIÓN ESCOLAR}

En el caso de Costa Rica, fue la Fundación Omar Dengo (FOD)², con el apoyo del Ministerio de Educación Pública (MEP), la institución que en el año 1988 impulsa el uso de la computadora y otros recursos tecnológicos en la educación primaria. El programa inicia con la dotación de

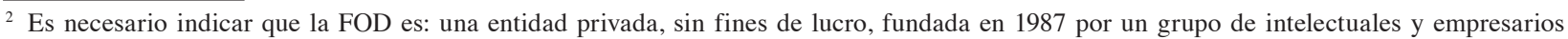
costarricenses que tiene como propósito promover el mejoramiento de la calidad de la educación costarricense, por medio de la introducción de nuevas tecnologías e innovaciones educativas. Consultado el 10 de junio de http://www.fod.ac.cr/programas/index.htm, junio 2006.
} 
infraestructura tecnológica (laboratorios de cómputo y programas informáticos específicos) en escuelas ubicadas en zonas urbanas socialmente vulnerables y en zonas rurales, en las cuales, por sus condiciones socioeconómicas, difícilmente los estudiantes tendrían posibilidad de interactuar con tales recursos.

Paralelamente, la Fundación promueve la capacitación de maestros sobre el uso de la computadora y el programa Logo en el aula.

El programa impulsado por la FOD se concibió desde sus orígenes como un proyecto de carácter nacional. Los objetivos actuales están dirigidos a contribuir a mejorar el sistema educativo del país; en particular, propiciar ambientes de aprendizaje que favorezcan en los estudiantes y en los educadores el desarrollo: del pensamiento lógico-matemático, de habilidades para la resolución de problemas como ampliación y profundización en temas curriculares, de la creatividad y de las actitudes positivas hacia el aprendizaje colaborativo. Lo mismo que el incremento de la autoestima y de la exploración de nuevos ambientes de aprendizaje mediante tecnologías digitales.

El programa “...parte de un marco filosófico constructivista, como fundamento epistémico y de un quehacer construccionista que orienta la práctica pedagógica” (Anfossi, Acuña y López, 2002, pp. 1-2).

Esta orientación pedagógica es la misma para todas las escuelas públicas, independiente de su ubicación geográfica, de sus características socioculturales o de sus necesidades específicas.

Con respecto al empleo de la Internet como recurso en los procesos educativos, el programa citado indica que los entornos de aprendizaje que diseñan, se caracterizan por cuatro principios:

- Enfatizan el aprendizaje: procuran el aprovechamiento del máximo potencial que ofrece internet como herramienta interactiva, hipermedial y de colaboración. Combinan, entonces, información y actividades educativas que los aprendices realizan e intercambian.

- Descentralizan el aprendizaje: propician múltiples entradas para el aprendizaje, de modo que benefician la promoción de diferentes estilos al aprender.

- Constituyen redes conceptuales: se presentan como conjuntos de nodos temáticos interrelacionados, que favorecen la transversalidad en el aprendizaje.

- Promueven la identidad y los valores universales: a través de los rasgos de una cultura de paz, el multiculturalismo, el respeto y disfrute de la diversidad, la actitud crítica y la creativa, se fomentan valores sustantivos para la construcción de una identidad sólida y tolerante (FOD, 2006).

Para lo anterior, se han impulsado acciones de desarrollo profesional y de aprendizaje permanente, dirigidas a atender las necesidades de capacitación de los maestros. Por lo que una de sus tareas prioritarias es identificar educadores “...interesados en aprender, en innovar metodologías, en explorar un paradigma educativo distinto, en conocer la tecnología y valorar su pertinencia pedagógica desde una práctica construccionista" (Anfossi y otros, 2002, p. 2).

Para concluir este apartado, es necesario indicar que una década después de haberse impulsado el Programa de la FOD, el Programa de Mejoramiento de la Calidad de la Educación Costarricense (PROMECE), introduce en la enseñanza secundaria la computadora y otros recursos tecnológicos, como apoyo curricular a los programas vigentes del MEP. La finalidad, al igual que el proyecto impulsado por la FOD, ha sido la formación de jóvenes constructores de conocimiento y no consumidores de información. 


\section{CARACTERÍSTICAS PRINCIPALES DE LAS REGIONES DE COSTA RICA Y SUS ESCUELAS}

Costa Rica se divide en seis regiones: Central, Chorotega, Pacífico Central, Brunca, Huetar Atlántica y Huetar Norte. Las regiones que tienen mayor población rural son: Brunca, Chorotega, Huetar Atlántica y Huetar Norte.

Cuadro 1

Distribución poblacional relativa por región, según el Censo 2000

\begin{tabular}{|l|c|c|c|c|c|c|c|}
\hline Zona & Central & Brunca & Chorotega & $\begin{array}{c}\text { Pacífico } \\
\text { Central }\end{array}$ & $\begin{array}{c}\text { Huetar } \\
\text { Atlántica }\end{array}$ & $\begin{array}{c}\text { Huetar } \\
\text { Norte }\end{array}$ & Total \\
\hline Urbana & 73 & 27 & 38 & 53 & 36 & 19 & 59 \\
\hline Rural & 27 & 73 & 62 & 47 & 64 & 81 & 41 \\
\hline
\end{tabular}

Fuente: INEC, 2001.

De acuerdo con el Censo del 2000, la región Pacífico Central tiene un porcentaje de población rural cercano al porcentaje nacional. Obsérvese en el cuadro 1, que la región Central es la que tiene el mayor porcentaje de población urbana, esto es, cerca de las tres cuartas partes de sus habitantes. Supera el promedio nacional en 14 puntos porcentuales.

De acuerdo con el XI Informe del Estado de la Nación en Desarrollo Humano Sostenible (Programa Estado de la Nación en Desarrollo Humano Sostenible, 2005b), el desarrollo de Costa Rica presenta desigualdades territoriales significativas, asociadas principalmente a la distribución de la población en el territorio nacional, a las distancias respecto a los centros donde se toman las decisiones, a la forma en como se ejecutan las políticas gubernamentales, a la manera en que se distribuye la inversión pública y privada, al tipo de actividades productivas que se desarrollan en cada región y, a la disponibilidades y uso de los recursos naturales en cada una de ellas. Pareciera que este desarrollo desigual, en lugar de superarse, tiende a profundizarse en algunas regiones.

Con respecto a los logros en desarrollo humano sostenible en el año 2004, el informe citado indica que "...Costa Rica ha entrado en una fase nueva y más peligrosa...", y "...ha empezado a "comerse su futuro", para mantener, en el corto plazo, la (apariencia de) normalidad..." (Programa Estado de la Nación en Desarrollo Humano Sostenible, 2005b, p. 45).

Según el Programa Estado de la Nación en Desarrollo Humano Sostenible (2005b), en cuanto al seguimiento del desarrollo humano por región, se afirma que durante el 2004:

Costa Rica sufrió un deterioro en las oportunidades para que su población tenga una mejor calidad de vida, que se expresó en un aumento a la pobreza, una reducción en los ingresos laborales de todos los estratos y una disminución del ingreso social que el Estado proporciona a los hogares...los más golpeados fueron los sectores sociales más pobres y, por tanto, más vulnerables... (p. 49).

Una de las mayores preocupaciones es que la pobreza en la Región Brunca, entre el 2003 al 2004, pasó de 33,6\% a 40,4\%. Lo mismo ocurrió en los otros indicadores relacionados con el 
empleo y seguridad social. En el cuadro 2, se presentan algunos indicadores sobre el desarrollo social, por región.

Cuadro 2

Algunos indicadores sociales al final del año 2004 (\%)

\begin{tabular}{|l|c|c|c|c|}
\hline Región & Tasa de desempleo & Hogares en pobreza & $\begin{array}{c}\text { Escolaridad } \\
\text { promedio }\end{array}$ & $\begin{array}{c}\text { Población de 12 a17 años } \\
\text { que asiste a educación }\end{array}$ \\
\hline Brunca & 5,8 & 40,4 & 6,4 & 73,9 \\
\hline Chorotega & 7,6 & 33,1 & 7,1 & 78,5 \\
\hline Huetar Atlántico & 6,0 & 23,6 & 6,4 & 72,9 \\
\hline Huetar Norte & 5,1 & 27,4 & 6,0 & 65,8 \\
\hline Pacífico Central & 7,1 & 25,6 & 6,7 & 72,0 \\
\hline Central & 6,6 & 17,1 & 8,7 & 82,9 \\
\hline
\end{tabular}

Fuente: Programa Estado de la Nación en Desarrollo Humano Sostenible. (2005b).

Se puede observar que durante el año 2004, la mayor tasa de desempleo se presentó en la región Chorotega, y la más baja en la región Central. El mayor porcentaje en pobreza se encontró en la región Brunca, y el más bajo, en la región Central. El mayor porcentaje de escolaridad se identificó en la región Central, y el más bajo, en la región Huetar Norte. Finalmente, el mayor porcentaje de la población de 12 años a 17 años dentro del sistema de educación formal, lo presentó la región Central, y el más bajo, la región Huetar Norte. En otras palabras, la región Central es la que presenta los indicadores más favorables en cada una de las variables consultadas.

Al consultarse el Informe del Estado de la Educación 1 (Estado de la Educación en Desarrollo Humano Sostenible, 2005a) y el estudio de Mora, R. y Ramos (2004) ${ }^{3}$, se constató que la brecha más significativa entre las zonas rurales y las zonas urbanas, se observa en la cobertura de la educación preescolar, en la cobertura del Programa del Informática Educativa FOD-MEP (PIE) y en la cobertura en la educación secundaria. En el cuadro 3 se destacan los porcentajes correspondientes, así como otros indicadores que muestran las diferencias entre las zonas urbanas y las rurales.

Cuadro 3

Brechas educativas en el año 2004 por zona (\%)

\begin{tabular}{|l|c|c|c|c|c|c|c|}
\hline Zona & $\begin{array}{c}\text { Niños (as) de } \\
\text { 3 a 5 años } \\
\text { que asisten a } \\
\text { preescolar }\end{array}$ & $\begin{array}{c}\text { Población de } \\
13 \text { a } 14 \text { años } \\
\text { que asiste a } \\
\text { educación }\end{array}$ & $\begin{array}{c}\text { Población de } \\
15 \text { a 17 años } \\
\text { que asiste a } \\
\text { educación }\end{array}$ & $\begin{array}{c}\text { Reprobación } \\
\text { en primaria }\end{array}$ & $\begin{array}{c}\text { Reprobación } \\
\text { en secundaria }\end{array}$ & $\begin{array}{c}\text { Cobertura PIE } \\
\text { en primaria }\end{array}$ & $\begin{array}{c}\text { Hogares } \\
\text { en pobreza }\end{array}$ \\
\hline Urbana & 55,8 & 93,8 & 78,9 & 8,1 & 21,2 & 71,8 & 15,4 \\
\hline Rural & 39,5 & 76,8 & 54,7 & 11,2 & 17,1 & 28,7 & 23,1 \\
\hline
\end{tabular}

Fuente: Programa Estado de la Nación en Desarrollo Humano Sostenible (2005a).

3 Se respeta el año de la referencia bibliográfica que aparece en el Informe del Estado de la Educación 2005 (b). No obstante, el documento que se logró acceder en Internet, aparece elaborado en el año 2002. 
En atención al precepto constitucional sobre la obligatoriedad de la educación preescolar, el MEP, fundamentado en la Ley 7676 del año 1997, pone en marcha en las Escuelas Unidocentes ${ }^{4}$ y en las Escuelas Dirección 1, dos acciones en pro de la cobertura en el nivel de preescolar. La primera consistió en nombramientos de Maestros Itinerantes de Preescolar, mediante los cuales se atendería niñas y niños de dos escuelas vecinas, con un promedio de matrícula de 20 alumnos en las dos instituciones. La segunda consistió en la conformación de Grupos Heterogéneos de estudiantes de Materno Infantil y Transición, los cuales serían atendidos por un docente de educación preescolar. En el 2003, solamente el 11\% de las escuelas unidocentes brindaba dicho servicio (Mora et al., 2002).

En el cuadro 3 se puede observar que el único indicador donde la zona rural obtuvo un mejor porcentaje, es en la reprobación en secundaria, lo que llama la atención. Las razones pueden ser de diferente índole; entre ellas, se podrían considerar la motivación o el compromiso de los estudiantes de estas zonas con el estudio, el tipo de distracciones que existen en estas zonas para los jóvenes, o, debido al incremento en los últimos años de la pobreza en las zonas urbanas. Solo un estudio dirigido a identificar tales razones podrá indicar, con la seriedad que ello implica, las razones que explican tal porcentaje.

El análisis por región muestra además que las regiones Huetar Norte y Brunca presentan los menores porcentajes de asistencia de la población infantil de cinco a seis años a la educación preescolar; por ejemplo, mientras para la región Central este porcentaje corresponde al 74,5\%, para las regiones Huetar Norte y Brunca es del 45,6\% y 59,3\%, respectivamente. Situación que más tarde incidirá en los resultados de aprobación en la enseñanza primaria (Mora et al., 2002).

Otros indicadores sobre el bienestar social, como la esperanza de vida, la mortalidad infantil y las coberturas educativas para el año 2005, no mostraron diferencias significativas por región, lo cual era previsible, dada la inversión del Estado en décadas anteriores, en dichas áreas.

En cuanto al estado del mobiliario de las escuelas de educación primaria, el Informe del Estado de la Educación 1, indica que en todas las regiones de Costa Rica se necesita mobiliario para la educación primaria. Por ejemplo, en el caso de los pupitres unipersonales, destacan algunas regiones donde se supera de manera significativa el porcentaje nacional de pupitres en mal estado (25,3\%), entre ellos, “35,3\% en Pérez Zeledón, 41,3\% en Upala, 55,6\% en Turrialba, 45,9\% en Nicoya, 46,6\% en Aguirre, 34,8\% en Limón y 38,1\% en Guápiles” (Programa Estado de la Nación en Desarrollo Humano Sostenible, 2005a, p. 45).

En el cuadro 4 se muestran algunos porcentajes sobre el rendimiento académico por zonas, según se indican en el Primer Informe de Seguimiento del Plan de Acción para la Educación para Todos (EPT) (Miniserio de Educación Pública, 2005). De acuerdo con este informe, las mayores diferencias porcentuales se presenta en repetición, tipo de institución (pública o privada) y en el número de estudiantes que trabajan.

Las escuelas públicas se clasifican por la matrícula en: Unidocente (Hasta 50 estudiantes), Dirección 1 (51-180 estudiantes), Dirección 2 (181-300 estudiantes), Dirección 3 (301-600 estudiantes), Dirección 4 (601-900 estudiantes) y Dirección 5 (Más de 900 estudiantes). Las Unidocentes y Dirección 1, se ubican por lo general, en zonas rurales. El resto, en zonas urbanas. Del total de escuelas en el 2005 (3707 escuelas), las Unidocentes representan cerca del $48 \%$ y las de Dirección 1, el 34\%; juntas cerca del 82\%. Mientras que la matrícula en conjunto (Unidocentes y Dirección 1) representa 32,5\% del total. 


\section{CUADRO 4}

Composición porcentual: Matrícula Educación Primaria 2005

por zona, repetición, sexo, dependencia y alumnos que trabajan

\begin{tabular}{|c|c|c|c|c|c|c|c|}
\hline \multirow{2}{*}{ Zona } & \multirow{2}{*}{$\begin{array}{c}\text { Porcentaje } \\
\text { de participación }\end{array}$} & \multirow{2}{*}{ Repetición } & \multicolumn{2}{|c|}{ Sexo } & \multicolumn{2}{|c|}{ Dependencia } & \multirow{2}{*}{$\frac{\text { Trabaja }}{2004}$} \\
\hline & & & Ho & M & Pública & Privada & \\
\hline Urbana & 56 & 6,7 & 51,2 & 48,8 & 87,6 & 12,4 & 1,3 \\
\hline Rural & 44 & 8,4 & 52,0 & 48,0 & 98,8 & 1,2 & 2,8 \\
\hline
\end{tabular}

Fuente: MEP. (2005).

Cuando se analizan los porcentajes de aprobación o de deserción para los años 2002, 2003 y 2004, por zona (5), se tiene que los porcentajes de aprobación de la zona urbana superan los de la zona rural. Situación contraria se da con los porcentajes de deserción.

\section{CUADRO 5}

Educación Primaria: porcentajes de aprobación y deserción por zona y año

\begin{tabular}{|l|c|c|c|c|c|c|}
\hline \multirow{2}{*}{ Zona } & \multicolumn{2}{|c|}{2002} & \multicolumn{2}{c|}{2003} & \multicolumn{2}{c|}{2004} \\
\cline { 2 - 7 } & Aprobación & Deserción & Aprobación & Deserción & Aprobación & Deserción \\
\hline \multirow{2}{*}{ Urbana } & 92,8 & 3,6 & 91,7 & 3,4 & 91,9 & 3,1 \\
\hline \multirow{2}{*}{ Rural } & 90,3 & 4,1 & 89,5 & 4,7 & 88,8 & 3,6 \\
\hline
\end{tabular}

Fuente: MEP. (2005).

Con respecto a las diferencias de jornadas escolares entre las escuelas rurales y escuelas urbanas, el estudio realizado por Mora et al. (2002) determinó que existen estudiantes de escuelas unidocentes que reciben hasta 240 horas menos de jornada escolar con respecto a las horas recibidas en escuelas urbanas. Este mismo estudio determinó, que el porcentaje anual nacional de deserción en los últimos 12 años fue de 4,5\%, mientras en las escuelas unidocentes alcanzó el 6\%. Asimismo, el porcentaje anual nacional de repitencia en los últimos 12 años fue cercano al 9,5\%, mientras que en las escuelas unidocentes fue de 11, $4 \%$.

Ahora bien, si se consideran los resultados del XII Informe Estado de la Nación (Programa Estado de la Nación en Desarrollo Humano Sostenible, 2006), sobre la población que completó cada ciclo de la educación regular, en el año 2005 mejoraron de manera significativa dichos porcentajes, con respecto a los obtenidos en los años 1994 y 1999. No obstante, siempre se observa una diferencia de 7 a 20 puntos porcentuales a favor de la zona urbana.

En el cuadro 6, se muestran estas cifras. 
Cuadro 6

Población que completa cada ciclo de la educación regular por zona

\begin{tabular}{|l|c|c|c|c|c|c|c|c|c|}
\hline \multirow{2}{*}{ Zona } & \multicolumn{3}{|c|}{1994} & \multicolumn{3}{c|}{1999} & \multicolumn{3}{c|}{2005} \\
\cline { 2 - 10 } & $\begin{array}{c}\text { I y II } \\
\text { Ciclos }\end{array}$ & $\begin{array}{c}\text { III } \\
\text { Ciclo }\end{array}$ & $\begin{array}{c}\text { XI } \\
\text { Año }\end{array}$ & $\begin{array}{c}\text { I y II } \\
\text { Ciclos }\end{array}$ & $\begin{array}{c}\text { III } \\
\text { Ciclo }\end{array}$ & $\begin{array}{c}\text { XI } \\
\text { Año }\end{array}$ & $\begin{array}{c}\text { I y II } \\
\text { Ciclos }\end{array}$ & $\begin{array}{c}\text { III } \\
\text { Ciclo }\end{array}$ & $\begin{array}{c}\text { XI } \\
\text { Año }\end{array}$ \\
\hline Urbana & 88,3 & 60,1 & 27,0 & 84,6 & 55,2 & 32,0 & 92,2 & 56,7 & 46,1 \\
\hline Rural & 74,1 & 27,4 & 7,0 & 74,2 & 31,4 & 11,6 & 85,6 & 39,1 & 28,0 \\
\hline
\end{tabular}

Fuente: Programa Estado de la Nación en Desarrollo Humano Sostenible. (2006).

Si se analiza el acceso a computadoras que tienen las instituciones educativas costarricenses, por zona, el estudio de Mora et al. (2002) determinó que el 60\% de escuelas y el $80 \%$ de colegios privados tienen disponibilidad a laboratorios de informática; mientras que solo el $20 \%$ de escuelas y el $60 \%$ de colegios públicos cuentan con un laboratorio de cómputo para uso de los estudiantes. En cuanto al acceso a la red Internet, se encontró que cerca del $60 \%$ de escuelas y colegios privados cuentan con esta posibilidad, y, en el caso de las instituciones públicas, solo el 20\% la poseen (Mora et al., 2002).

En el cuadro 7, se presentan por zona, algunos porcentajes sobre el acceso que tuvieron los hogares costarricenses en los años 2000, 2001 y 2003 a computadoras y a Internet. Obsérvese que los porcentajes difieren de manera significativa por región. Por ejemplo, en el año 2003, el 29\% de los hogares de zonas urbanas tenían acceso a una computadora, y cerca del 13\% a Internet. Mientras que para ese mismo año, solo el $10 \%$ de los hogares de zonas rurales tenían acceso a computadora y cerca del $3 \%$ a Internet.

\section{CUADRO 7}

Acceso a computadora e Internet en los hogares costarricenses

\begin{tabular}{|c|l|c|c|c|}
\hline \multirow{2}{*}{ Zona } & Acceso al recurso en porcentaje & 2000 & 2001 & 2003 \\
\hline \multirow{2}{*}{ Urbana } & Computadora & 19,64 & 23,83 & 29,30 \\
\cline { 2 - 5 } & Internet-total (teléfono o cable) & 6,11 & 7,81 & 13,42 \\
\hline \multirow{2}{*}{ Rural } & Computadora & 5,31 & 7,51 & 10,59 \\
\cline { 2 - 5 } & Internet -total (teléfono o cable) & 0,90 & 1,56 & 3,13 \\
\hline
\end{tabular}

Fuente: Con base en el Cuadro A.1, Anexos Estadísticos. (Monge, R. y et al., 2004).

Al analizar los porcentajes de hogares con acceso a computadora y a Internet en el año 2003, por región (8), se tiene que la región Central es la que presenta los mayores valores en ambos casos. La región Huetar Atlántica presenta los porcentajes menores. Las otras regiones, a pesar de que presentan porcentajes mayores a esta última, difieren significativamente de los valores de la región Central. 
Cuadro 8

Acceso a computadora e Internet en el hogar en el 2003, según región

\begin{tabular}{|c|c|c|c|c|c|c|}
\hline $\begin{array}{c}\text { Acceso al recurso en } \\
\%\end{array}$ & Central & Chorotega & $\begin{array}{c}\text { Pacífico } \\
\text { Central }\end{array}$ & Brunca & $\begin{array}{c}\text { Huetar } \\
\text { Atlántico }\end{array}$ & $\begin{array}{c}\text { Huetar } \\
\text { Norte }\end{array}$ \\
\hline Computadora & 28,56 & 11,11 & 10,09 & 10,53 & 6,94 & 13,13 \\
\hline Internet-Total & 12,93 & 2,86 & 2,76 & 2,73 & 2,02 & 5,16 \\
\hline
\end{tabular}

Fuente: Con base en el Cuadro A.2, Anexos Estadísticos de Monge, R. et al. (2004).

Si se centra la atención en el acceso a computadora y a Internet en los hogares, según el nivel educativo del jefe (a) de hogar (9), se puede observar que el acceso a ambos recursos está asociado al nivel educativo del jefe o jefa de hogar.

Cuadro 9

Acceso a computadora e Internet en el hogar, año 2003, según nivel educativo del jefe (a) de hogar

\begin{tabular}{|c|c|c|c|c|}
\hline $\begin{array}{c}\text { Acceso al recurso } \\
\text { en } \%\end{array}$ & Ninguno & Primaria & Secundaria & Universitaria \\
\hline Computadora & 2,06 & 8,66 & 25,68 & 61,99 \\
\hline Internet-total & 0,00 & 2,20 & 7,44 & 36,87 \\
\hline
\end{tabular}

Fuente: Con base en el Cuadro A.13, Anexos Estadísticos. (Monge, R. et al., 2004).

Para el 2003, en el cuadro 10, se presenta el acceso y uso a Internet por zona, por miembro de familia, y si el hogar tenía o no conectividad.

Nótese en el cuadro 10, que la diferencia por tipo de hogar, con o sin conectividad a Internet, por miembro de familia, es poca. Se puede afirmar que en ambos casos, los porcentajes son cercanos, inclusive en la categoría "hijos". Lo interesante es comprobar que son los hijos, y no los progenitores, quienes presentan los mayores porcentajes de uso del Internet. Además, que cuando los hogares no tienen conectividad a Internet, independientemente de si estos se ubican en zonas urbanas o rurales, la categoría "Café Internet" es la que presenta los mayores porcentajes para acceder a este recurso. En segundo lugar, se ubica la categoría denominada "el lugar de trabajo".

En cuanto al uso que se le da a Internet por familia, se puede comprobar que las categorías "Búsqueda de información" y "Correo electrónico", son las que presentan los mayores valores para los hogares que se ubican en zonas urbanas como en rurales. En el tercer lugar, se ubica la categoría "Chatear", en ambos casos. 


\section{Cuadro $\mathrm{N}^{\mathrm{o}} 10$}

Acceso, uso de Internet por los miembros del hogar, con o sin conectividad

\begin{tabular}{|c|c|c|c|c|c|}
\hline & \multirow{2}{*}{ Año 2003} & \multicolumn{2}{|c|}{ Hogar con conectividad } & \multicolumn{2}{|c|}{ Hogar sin conectividad } \\
\hline & & Urbana & Rural & Urbana & Rural \\
\hline \multirow{3}{*}{ ¿Quién? } & Cónyuge & 46,32 & 42,84 & 18,77 & 15,58 \\
\hline & Hijos & 71,38 & 61,19 & 63,41 & 67,66 \\
\hline & Total familias con un usuario & 38,02 & 47,12 & 76,09 & 84,66 \\
\hline \multirow{4}{*}{ ¿Dónde? } & Centro educativo & 26,86 & 20,98 & 28,64 & 26,25 \\
\hline & Café Internet & 14,00 & 18,80 & 58,60 & 58,25 \\
\hline & Lugar de trabajo & 40,09 & 37,49 & 43,51 & 36,38 \\
\hline & $\begin{array}{l}\text { Total familias que usan } \\
\text { Internet en el mismo lugar }\end{array}$ & 43,90 & 44,02 & 68,94 & 73,51 \\
\hline \multirow{4}{*}{ ¿Usos? } & Correo electrónico & 82,78 & 72,21 & 62,39 & 54,70 \\
\hline & Búsqueda de información & 92,34 & 91,62 & 87,58 & 90,86 \\
\hline & Chatear & 30,72 & 27,47 & 24,75 & 19,62 \\
\hline & $\begin{array}{l}\text { Total familias que hacen solo } \\
\text { una cosa con Internet }\end{array}$ & 12,49 & 20,49 & 35,66 & 43,63 \\
\hline
\end{tabular}

Fuente: Con base en el Cuadro A.6, Anexos Estadísticos. (Monge, R. et al., 2004).

En el cuadro 11 se presentan los porcentajes de cobertura por zona del Programa de la FODMEP, en enero del 2005. Como se puede constatar, la cobertura del Programa citado en las escuelas rurales en cada provincia, es baja. Los mayores porcentajes se ubican en San José, Cartago y Heredia. Nótese además, que el porcentaje de cobertura en zonas rurales en el nivel nacional, no llega ni al 30\%. Más aún, el porcentaje de las zonas rurales que pertenecen a Puntarenas y Alajuela, está por debajo del promedio nacional.

Cuadro 11

Cobertura (\%) del Programa FOD en Escuelas por provincia y zona

\begin{tabular}{|l|c|c|}
\hline Provincia & Urbana & Rural \\
\hline San José & 75,6 & 30,8 \\
\hline Alajuela & 73,2 & 27,1 \\
\hline Cartago & 68,6 & 37,5 \\
\hline Heredia & 78,0 & 38,0 \\
\hline Guanacaste & 64,4 & 30,0 \\
\hline Puntarenas & 53,7 & 19,6 \\
\hline Limón & 67,3 & 28,8 \\
\hline TOTAL & 71,8 & 28,7 \\
\hline
\end{tabular}

Fuente: FOD. (2005). 
Cuando se analiza la cobertura del Programa de la FOD-MEP con conectividad a Internet, se tiene que en el año 2001 (12), del total de escuelas participantes en dicho programa, solo al 5\% se le otorgó acceso a Internet.

Cuadro 12

Escuelas en el Programa FOD, por matrícula, con conectividad por zona

\begin{tabular}{|l|c|c|c|c|}
\hline \multirow{2}{*}{ Matrícula 2001 } & \multicolumn{2}{|c|}{ Urbanas } & \multicolumn{2}{c|}{ Rurales } \\
\cline { 2 - 5 } & Absoluto & $\%$ & Absoluto & $\%$ \\
\hline $101-250$ & 2 & 1,58 & 5 & 6,32 \\
\hline $251-500$ & 15 & 11,9 & 28 & 35,44 \\
\hline $501-1000$ & 74 & 58,73 & 37 & 46,83 \\
\hline Más de 1001 & 35 & 27,78 & 9 & 11,39 \\
\hline TOTAL & 126 & 61,46 & 79 & 38,53 \\
\hline
\end{tabular}

Fuente: Con base en el Cuadro 3. (Monge R. et al., 2004).

Nótese, en el cuadro 12, que del porcentaje de escuelas con conectividad, cerca del $39 \%$ corresponde a escuelas rurales. Se debe recordar que el mayor porcentaje de escuelas en las zonas rurales, lo constituyen las escuelas Unidocentes y las de Dirección 1. Este grupo de instituciones representaba en el 2001 más de las dos terceras partes del total de escuelas del país. No obstante, a las escuelas Unidocentes ${ }^{5}$ que participaban en el programa de la FOD, no se les otorgó acceso a Internet y, de las clasificadas como Dirección 1, solamente a 5.

En resumen, el programa de la FOD, en el año 2001 -aproximadamente 12 años después de su puesta en marcha-, está casi totalmente ausente en la "típica escuela rural" costarricense. Más aun, pareciera que el programa sólo se ejecuta en las escuelas rurales ubicadas en las zonas de mayor concentración poblacional.

Con respecto a la universalización del programa de informática educativa en la educación primaria, supuestamente con énfasis en las comunidades rurales, el Primer Informe de Seguimiento del Plan de Acción de la EPT (Ministerio de Educación Pública [MEP], 2005, p. 134), indica que en el año 2004, “...65 escuelas Unidocentes fueron dotadas de nuevos equipos y accesorios (31 de ampliación y 34 de renovación)". El mismo informe argumenta que la falta de acceso a la tecnología en las escuelas rurales se debe a varias razones, en particular:

- La ubicación geográfica de las escuelas.

- $\quad$ El difícil acceso que tienen a la energía eléctrica.

- $\quad \mathrm{Al}$ hecho de que estas escuelas requieren de una orientación pedagógica diferente, la cual se ha venido construyendo en los últimos seis años que lleva en vigencia el Proyecto: "La computadora en el aula" en 59 escuelas Unidocentes.

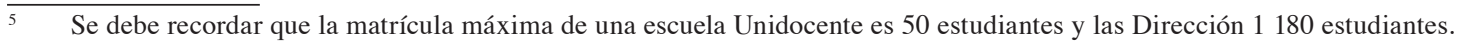


Por lo tanto, se concluye que los logros sobre la universalización del programa de informática educativa en la educación primaria rural, en el año 2004, son mínimos.

En cuanto a los requerimientos de centros de informática para la educación primaria, el Informe del Estado de la Educación 1, afirma que se presentan en todas las regiones, pero, particularmente en: "Puriscal, Alajuela, Pérez Zeledón, San Carlos, Upala, Turrialba, Nicoya, Coto y Guápiles, donde la necesidad del recurso adicional duplica o triplica el existente" (Programa Estado de la Nación en Desarrollo Humano Sostenible, 2005a, p. 45).

\section{¿QUÉ INDICAN LAS POLÍTICAS CON RESPECTO A LA EDUCACIÓN RURAL Y LAS TIC?}

Al pasar al plano de las políticas educativas, se puede comprobar que en los tres planes gubernamentales $^{6}$ en vigencia, se visualiza la educación rural como una de las prioridades. De hecho, los tres planes son bastante coherentes en términos de "políticas", "ejes principales" o "estrategias"; términos que utilizan para referirse a las orientaciones o lineamientos en cada área, y al fortalecimiento de la educación rural.

Por ejemplo, en el documento "Costa Rica Marco Institucional y contexto" en la sección Plan Educativo 2002-2006 y apartado Política y acciones estratégicas; la Política 2 "Mejoramiento de la calidad del servicio en la Educación Primaria, con énfasis en las comunidades más desfavorecidas" (p. 8), se indica la necesidad de fortalecer las escuelas indígenas, las escuelas unidocentes y las escuelas ubicadas en zonas de menor desarrollo social. Lo mismo sucede con la Política 7 "Garantizar a la población escolar y estudiantil en condiciones de pobreza y pobreza extrema, los beneficios de los Programas de Equidad" (p. 10), en la Política 8 "Mejoramiento de la condición de los docentes" (p. 11), en la Política 9 "Mejoramiento de las condiciones de Infraestructura, Mobiliario y Materiales Educativos" (p. 11), en la Política 11 "Incorporación en los procesos y contenidos educativos las diferentes perspectivas del desarrollo humano" (p. 12) y, en la Política 15 "Universalización del Programa Nacional de Informática Educativa” p. 15) (MEP, s.f., pp. 8-10-1112-14).

En el Plan de Relanzamiento de la Educación Costarricense , el objetivo 2 indica "Posicionar a la educación costarricense, como eje prioritario en la agenda social del país, en el marco del combate a la pobreza, la reducción de las brechas entre la educación rural y la educación urbana, concurriendo con equidad" (MEP, 2004a). Para el cumplimiento de este objetivo se establecen cuatro prioridades, entre ellas la educación rural.

En cuanto al Plan de Acción para la Educación Para Todos (Mep, 2004b), el cual se refiere a los compromisos asumidos por Costa Rica en Dakar, Senegal, en el 2000 -con respecto a la educación que tienen derecho todos los ciudadanos independiente de su edad, género u origen étnico-, se puede constatar que una de las prioridades mencionadas es la educación primaria rural, lo cual también se infiere del Informe de Seguimiento del Plan de Acción para la EPT (MEP, 2005).

En síntesis, en distintos documentos oficiales del MEP, se afirma que la educación rural es una de las prioridades de las políticas gubernamentales. No obstante, los datos comentados en el

Plan educativo 2002-2006, Plan para el Relanzamiento de la Educación Costarricense y, Plan de Acción de la Educación para Todos (EPT) 2003-2015. 
presente artículo evidencian una distancia significativa entre el discurso y la realidad. Pareciera todo lo contrario, se trata de uno de los sectores más abandonados por los gobiernos de turno.

\section{LOGROS: PROGRAMA DE LA FOD Y PROGRAMA DE INFORMÁTICA EN SECUNDARIA}

A pesar de los esfuerzos que ha realizado el MEP en conjunto con la FOD en la enseñanza primaria y el Programa PROMECE, en la enseñanza secundaria; en particular en dotación de infraestructura tecnológica y en capacitación de docentes sobre el uso de las TIC en la educación, aún falta mucho por hacer con respecto a la cobertura e impacto de ambos proyectos. El Informe del Estado de la Educación (2000) afirma, en relación con el Programa de la FOD:

En 1990 la cobertura de este programa en la educación primaria y preescolar públicas era de 22,1\% y se mantuvo alrededor de un 24\% hasta 1997; su impacto comenzó a aumentar de manera significativa a partir de 1998, con un $37,4 \%$, hasta alcanzar en el 2004 el 53,1\%, lo cual representaba una cifra absoluta de 306.217 estudiantes beneficiados (...). La cobertura por zona geográfica en el mismo nivel educativo es de $71,8 \%$ en la zona urbana y de $28,7 \%$ en la rural... (p. 59).

Al referirse al Programa para Secundaria, el mismo informe indica que en el tercer ciclo, la cobertura en el 2004 fue de apenas el 72,8\%. Luego, agrega que la Contraloría General de la República (CGR), mediante un estudio realizado por el Programa de Informática de Secundaria “...planteó una serie de observaciones, entre las cuales se encuentra la falta de una adecuada integración al proceso educativo"... (Programa Estado de la Nación en Desarrollo Humano Sostenible, 2005a, p. 60). A continuación, se transcriben las observaciones respectivas de la CGR citado por el (Programa Estado de la Nación en Desarrollo Humano Sostenible, 2005a):

En un estudio efectuado en el programa de Informática Educativa para Secundaria (PRIES) en el año 2002, se determinó que la falta de un programa estandarizado, aprobado por el Consejo superior de Educación, que establezca un mínimo de conocimiento y desarrollo esperados de los educandos, así como la débil contribución de los profesores de materias básicas, debilitan las posibilidades del apoyo curricular que pretende el programa, según uno de los objetivos específicos (p. 60).

Con respecto a la evaluación social participativa de la educación rural, el Informe mencionado indica que el estudio de la CGR, al referirse al Programa de la FOD, “...llega a conclusiones más positivas, aunque se señala cierta insuficiencia en la amplitud del programa en las escuelas multigrado (unidocentes y Dirección 1)" (Programa Estado de la Nación en Desarrollo Humano Sostenible, 2005a, p. 60). Dada la importancia con la temática discutida, a continuación se transcriben las conclusiones relacionadas con los docentes:

Los docentes valoran en forma muy positiva este proyecto, especialmente por el impacto que ha tenido en los alumnos; incluso afirman que los niños se sienten más motivados por la escuela desde que tienen ese proyecto informático (...). Los docentes 
reconocen que la capacitación que han recibido ha sido muy pertinente. Las limitaciones que se perciben es que una sola computadora a veces no llena las necesidades ni las expectativas. Asimismo, el docente tiene poco tiempo para apoyarlos enseñándoles lo que él ha aprendido en la capacitación (DNI, 2003b) (Programa Estado de la Nación en Desarrollo Humano Sostenible, 2005a, p. 60).

En el contexto nacional destacan además los resultados del estudio realizado por Monge y Hewitt (2004) sobre el acceso y uso de las computadoras y la Internet, por los hogares costarricenses y en la educación primaria, durante los años 2000, 2001 y 2003. De acuerdo con estos autores, la brecha digital -entendida como el acceso diferenciado que tienen las personas a las TIC, “...así como las diferencias en la habilidad para usar tales tecnologías, el empleo (...) que le dan a éstas y en el impacto que el uso tiene sobre el bienestar" (Monge et al., 2004, p. XIII)-, en el caso costarricense, continúa siendo un desafío, pues con el estudio realizado, se comprobó que dicha brecha “...se sigue mostrando con claridad entre los hogares de diferentes zonas geográficas, de disímiles rangos de ingreso y de diversos niveles de educación” (p. XIII).

Con respecto a la cobertura de Internet, se constató que durante el período 2000 al 2003 inclusive, el incremento anual es de 1,8 puntos porcentuales. Por lo tanto, si se mantiene ese incremento, Costa Rica requeriría de 45 años para alcanzar una cobertura total del servicio de Internet.

El estudio muestra además, que "...las escuelas de mayor población estudiantil, ubicadas en las zonas urbanas y de mayores niveles de ingreso, son las que tienen acceso a las computadoras y, en algunos casos a la Internet." (Monge et al., 2004, p. XIV). De donde que los niños y las niñas de las zonas más alejadas y de menor ingreso, así como las escuelas unidocentes, no tienen acceso a las TIC.

El estudio realizado concluye, refiriéndose al sistema educativo costarricense:

...Tal parece que contamos con un sistema educativo muy distante de desempeños y logros; con baja eficiencia y eficacia, especialmente secundaria; con desiguales oportunidades para poblaciones de zonas rurales respecto a urbana, resto del país y zonas periféricas...(Mora, R. et al., 2002, p. 87).

Con lo cual sugieren la necesidad de una reforma educativa de consenso, “...que trace los grandes derroteros educativos del país para los próximos veinte años y que trascienda actos de gobierno..." (Mora et al., 2002, p. 88).

Como un aporte del estudio realizado, se identifican siete desafíos para el Sistema Educativo costarricense. Para cada uno se establecen diferentes componentes estratégicos, todo con la finalidad de definir algunas orientaciones para avanzar en el diseño de una política de Estado en materia de Educación y Conocimiento, claro está, con una amplia participación de los sectores de la sociedad civil.

A continuación, se cita el Desafío 2, el componente estratégico V relacionado con la temática de interés del presente artículo y algunos principios orientadores del componente, con el propósito de mostrar la claridad de los autores con respecto al uso de las TIC en el medio rural:

Desafío 2: Brindar herramientas que permitan el pleno ejercicio de la ciudadanía y acceder a conocimientos y habilidades que permitan una incorporación exitosa al mundo del trabajo... 
V. Integrar las tecnologías de información y comunicación al sistema educativo del país (público y privado) y cerrar la brecha digital...

En este componente debe quedar claro:

- El papel que van a jugar las tecnologías de información y conocimiento en el procesos de enseñanza-aprendizaje de nuestros niños, niñas y adolescentes.

- El papel que van a jugar las tecnologías de información y conocimiento en la función de enseñanza de los maestros y profesores al impartir lecciones.

- El compromiso por incorporar las tecnologías de información y comunicación en el proceso educativo de las niñas, niños y adolescentes de nuestro país, y cómo hacer partícipes a los educadores para que amplíen sus posibilidades pedagógicas con sus alumnos.

- Si va considerar fundamental integrar las tecnologías de información y comunicación como una herramienta habitual en el proceso de enseñanza-aprendizaje y cómo garantizar que todos los niños, niñas y adolescentes del país tengan acceso y se familiaricen con el uso de medios de información como Internet y las computadoras personales.

- El compromiso para garantizar que los centros educativos públicos cuenten con la infraestructura; los equipos, la actualización y la renovación de los mismos; la formación y actualización docente necesarias; los contenidos educativos necesarios; los servicios de comunicación necesarios para incorporar e integrar las tecnologías de información y comunicación al proceso de enseñanza-aprendizaje.

- La política del país para mejorar e invertir en infraestructura de telecomunicaciones necesaria, que garantice poner al servicio de todos los centros educativos medios tecnológicos novedosos que contribuyan en la formación de los alumnos y en mejorar la calidad de la educación y, especialmente, garantizar el acceso y uso de estos medios a las poblaciones más alejadas del país y a los de menores ingresos.

Lamentablemente, propuestas como las anteriores no son escuchadas por los entes o autoridades gubernamentales que toman las decisiones. Por lo que es previsible que las interrogantes planteadas permanezcan en la mesa de discusión.

\section{OTROS ESTUDIOS QUE PUEDEN CONTRIBUIR AL ANÁLISIS DE LA TEMÁTICA}

En el contexto internacional, es necesario considerar los resultados del estudio realizado por Arredondo, Catalán, Montesinos y Monsalve, sobre la introducción de las TIC en dos escuelas de Chile. La investigación se orientó por preguntas tales como:

... ¿Esta tecnología se incorpora realmente como una nueva herramienta pedagógica a la escuela? ¿Qué aspectos culturales se producen en el ámbito rural-local a partir de la introducción de esta nueva tecnología globalizada? ¿Qué sucede en la relación profesoralumno en la escuela rural cuando se introducen las tecnologías de la información y las innovaciones pedagógicas que estas conllevan? ¿Cómo reacciona el alumno frente a este nuevo recurso educativo? (Arredondo, Catalán, Montesinos y Monsalve, 2001, p. 6). 
El interés principal era contrastar la concepción del uso de tales herramientas en los objetivos del programa y el enlace con la práctica que se lleva a cabo en las escuelas rurales que participan en este. Además, evidenciar la necesidad de cambiar tanto la concepción pedagógica del programa Enlace, así como la práctica educativa de este en el aula, con la finalidad de que dichas herramientas efectivamente posibiliten la superación de la pobreza en las zonas rurales de Chile.

Del análisis realizado por los investigadores concluyen que la percepción:

... que se tiene en Chile y que reproduce el proyecto Enlaces, para superar este problema [pobreza], generalmente, está orientada en una perspectiva estrictamente cuantitativa, que señala que todo este retraso pasa por la poca cobertura tecnológica, la que se refleja, por ejemplo, en la insuficiente cantidad, de computadores por número de habitantes y la aún escasa conexión a Internet (Arredondo, 2001, p. 28).

Y agregan más adelante:

...la única forma de cerrar efectivamente la brecha digital es a través de la creación de un vasto proceso de "alfabetización tecnológica" proceso que facilite en los usuarios el desarrollo de una "cultura tecnológica" que supere el mero uso técnico (...) nos parece oportuno plantear un tema que debe empezar a ser debatido para optimizar la introducción de las TIC y así aproximarse a disminuir la "brecha digital". Este tema es el de analizar los supuestos de base sobre los que se sustentan programas como los de la Red Enlaces, y la ambigüedad con que estos objetivos finales son planteados... (Arredondo, 2001, p. 29).

Otro estudio que merece ser considerado en el análisis del uso de las TIC en el medio rural, es el realizado por Marchesi y Martín. La indagación realizada por estos investigadores trata de responder a tres interrogantes:

¿Aunque solo cambiara los sistemas de representación de la información y no se alterara el modelo educativo, sería positivo utilizar las TICs? ¿Favorecen las TICs la transformación del modelo de enseñanza de los profesores? ¿Existen algunas condiciones que favorecen la incorporación de las TICs en la enseñanza y otras que lo desaconsejan? (Marchesi y Martín, 2003, p. 56).

Los objetivos del estudio estaban dirigidos a determinar cambios en las creencias y actitudes de los profesores y de los estudiantes con respecto a las TIC, y sus efectos en la motivación, interés y en la valoración que tienen sobre la enseñanza y el aprendizaje; así como comprobar el impacto de las TIC en las relaciones entre los estudiantes y a identificar los aspectos que obstaculizan o facilitan el uso de las TIC.

Entre los resultados obtenidos destacan los siguientes:

- La mayoría de los docentes considera que les va a ser más difícil mantener el orden en el aula de informática.

- Algunos profesores valoran positivamente la flexibilidad metodológica que se da al trabajar con las TIC, otros creen que lo más positivo es el ambiente de trabajo que se genera en los alumnos. 
- Los resultados analizados sugieren que la enseñanza con ordenador no cambia por sí misma los modelos de enseñanza o de aprendizaje de los profesores, sino que, más bien, se tiende a adaptar el uso de las TIC al modelo educativo del docente.

- La experiencia sí modifica algunas creencias y expectativas de los docentes, pero no lo suficiente para cambiar los significados que tiene cada docente sobre cómo enseñar y cómo aprender.

- $\quad$ Pareciera que las concepciones de los profesores sobre los procesos de enseñar y aprender y sobre su forma de evaluar, pueden estar fuertemente arraigados, lo que es difícil modificarlos con experiencias que duran dos o tres meses.

- $\quad$ Pareciera que lo ideal hubiese sido articular, desde el inicio, el diseño de las unidades temáticas con el uso de las TIC, por ejemplo, mediante el desarrollo de un proyecto a través de Internet, para valorar el impacto de las TIC tienen en las transformaciones del modelo educativo que por general, aplica el docente.

Los dos resultados más interesantes del estudio fueron, primero, comprobar que conforme los docentes utilizan más las TIC, menos expectativas tienen sobre ellas. El segundo, no encontrar una diferencia significativa, en términos estadísticos, entre los resultados académicos de los estudiantes que utilizaron las TIC y los que no las usaron.

\section{¿CUÁLES SON LAS LECCIONES APRENDIDAS CON LA REFLEXIÓN REALIZADA?}

En primer lugar, hacemos nuestras las palabras de Tedesco, J. (2003), cuando considera que para integrar de manera efectiva las TIC en un proyecto destinado a reducir las desigualdades, es preciso que tales recursos:

Formen parte de un modelo pedagógico en el cual los componentes que han sido identificados como cruciales para romper el determinismo social sean asumidos por los procesos que impulsan las tecnologías. Esto tiene que ver, obviamente, con los contenidos, pero también con los métodos. En este sentido, no es banal ni reiterativo insistir en que la prioridad debe ser puesta en los docentes.

Por lo tanto, es necesario que los docentes de las escuelas rurales comprendan que las TIC por sí solas no contribuyen a mejorar los procesos educativos escolares. Además, que el uso de cualquier recurso (digital o no), de manera aislada, sin los objetivos de aprendizaje pertinentes o apropiados, sin una estrategia metodológica deliberada, la cual considere las necesidades educativas, expectativas e intereses de los estudiantes y sus comunidades, carece de sentido.

Las TIC se constituyen en un recurso más, pero "muy poderoso" cuando se utilizan de manera apropiada. Y, como todo recurso didáctico tiene sus limitaciones y desventajas.

En segundo lugar, de acuerdo con los datos analizados, se puede asegurar que un alto porcentaje de niñas y niños de escuelas rurales costarricenses no tienen acceso a estos recursos, lo cual los deja en desventaja con respecto a la formación que reciben niños y niñas de escuelas urbanas o de mayores ingresos familiares. Esta inequidad es preocupante, porque existen experiencias concretas, por ejemplo, en Perú, donde se ha comprobado, mediante proyectos pilotos, que “...las TIC, si son integradas de manera adecuada a esfuerzos de desarrollo rural, pueden tener un impacto importante" (Bossio, López, Saravia y Wolf, 2004, p. 1) en dichas regiones.

En tercer lugar, es un hecho que el problema de la pobreza y de las inequidades sociales no se soluciona mediante una transformación educativa y la incorporación de las TIC en los salones 
escolares. Sin embargo, se han utilizado tales argumentos tanto en el contexto nacional como en el internacional, como la respuesta a la urgente necesidad de adecuar la educación a los procesos de mundialización de la economía, que se impulsan en el entorno internacional.

Lo preocupante es que no se analizan los mensajes implícitos de dicho discurso, como sacralizar las TIC, y lo que es peor, sugerir que estos recursos actúan por sí solos. Por lo tanto, el no obtener provecho de ellos es una responsabilidad exclusiva de los usuarios.

La gran interrogante es: ¿para qué utilizar las TIC en las escuelas rurales?

De lo expuesto en los apartados anteriores, se infiere la "...cruda realidad" que enfrentan cotidianamente la mayoría de las escuelas rurales en Costa Rica. Además, que en la actualidad, lo rural no es sinónimo de producción agrícola, forestal..., sino que debe concebirse como un espacio donde habitan personas que además de ocuparse en estas y otras actividades, se esfuerzan por una acción más integral de desarrollo. En algunos casos, en dichas zonas coexisten actividades agrícolas con otras alternativas económicas. Pero además, las nuevas generaciones saben, por diferentes medios, que afuera existen otras opciones de desarrollo personal, familiar o comunal, que distan de las que ellos vivencian y, quiérase o no aceptar, reclaman por tales oportunidades.

Por lo tanto, el no brindar nuevas oportunidades de desarrollo personal o familiar, es impulsar a las generaciones más jóvenes a emigrar a otros sitios donde pueden satisfacerlas con el peligro de que el capital sociocultural que ha caracterizado a estas comunidades se debilite.

Por otra parte, desde nuestra óptica, una de las razones que motivan las observaciones del estudio de la CGR al Programa que desarrolla la FOD y al Programa de Informática en Secundaria, es precisamente impulsar en ambos el uso de las TIC en los procesos educativos, al margen de la cotidianidad que vivencian los estudiantes y las necesidades educativas de las comunidades.

En este sentido, se considera que si el uso de las TIC en los salones escolares respondiera a tales necesidades educativas, la participación de las comunidades en los procesos escolares se incrementaría. Consecuentemente, los logros redundaran en beneficio de los mismos involucrados, al existir un mayor interés de la comunidad en la pertinencia y calidad de los procesos educativos que se ofrecen en las escuelas.

Es un hecho que si se modifica el fundamento pedagógico del uso de las TIC en los procesos educativos escolares, por un enfoque que apoye el desarrollo de determinadas competencias o habilidades en los estudiantes, en pro de una formación de niños, niñas y jóvenes emprendedores, las posibilidades de desarrollo de las zonas rurales serían mayores.

Esto no significa que solo mediante el uso de las TIC dichas competencias se logran. El reto está en elaborar una propuesta pedagógica deliberada que justifique las inversiones que viene realizando el país en el área tecnológica, bajo el discurso de la "innovación de los procesos educativos en la Educación General Básica". Desde nuestra perspectiva, mientras no se modifique el enfoque sobre el uso de las TIC en los procesos educativos, de manera que parta del contexto sociocultural y vivencial en la cual están inmersas las escuelas rurales, la inversión que se haga para dotar a estas instituciones de infraestructura tecnológica, corre el riesgo de perderse. Lo cual no se justifica en un país donde las desigualdades se incrementan cada vez más.

Es un hecho que si se contara con una propuesta pedagógica para las escuelas rurales, donde se integren los procesos educativos, la tecnología y el desarrollo de las comunidades respectivas, se incrementarían las posibilidades de desarrollo de los estudiantes y de las comunidades.

Ahora bien, desde nuestra óptica, para ello se requiere vincular, al menos, cinco componentes que cada comunidad debe poseer: 
1. Un Proyecto de Desarrollo Local construido con la participación activa de todos los miembros de la comunidad (niños, niñas, jóvenes, adultos, mujeres, varones y adultos mayores, incluyendo los educadores y los involucrados con los procesos educativos).

2. El Proyecto de Desarrollo Local debe considerar la generalización de alternativas de empleo o autoempleo, que, por un lado, diversifiquen la economía local con la finalidad de desalentar la dependencia económica en el uso de los recursos naturales; y por otro, propicien la gestión de proyectos productivos de emprendimiento familiar o grupal.

3. Una propuesta curricular que responda a las necesidades educativas de la comunidad. Esta propuesta además de ser coherente con el Proyecto de Desarrollo Local, debe ser amplia y flexible. En este sentido debe, considerar opciones tanto en el área de la educación formal como de la educación no formal. Pero sobre todo, impulsar el desarrollo de proyectos pedagógicos que propicien en los estudiantes (niños, jóvenes y adultos) aptitudes de emprendimiento, de manera que se promueva la formación de un ciudadano creativo, reflexivo, analítico y emprendedor.

4. Servicios básicos de salud que complementen las acciones anteriores.

5. La satisfacción de las necesidades alimentarias de los habitantes.

Obviamente, lo anterior implica una transformación de los procesos educativos en la escuela rural, los cuales se ejecutan en función de las directrices emanadas de las oficinas centrales. Consecuentemente, implicaría una transformación de la gestión de las Direcciones Regionales del MEP, de los Directores de las Instituciones educativas, de la relación escuela-familia-comunidad, y una mayor coordinación entre el quehacer que realizan las distintas instancias gubernamentales relacionadas con el desarrollo de las comunidades rurales con los gobiernos locales.

En síntesis, si tales cambios fueran posibles, la mayor contribución que podría hacer la educación rural al desarrollo de la comunidad es transformarse en una opción viable, cuya finalidad es la formación de niños, niñas, jóvenes y adultos emprendedores, creativos, comprometidos con la riqueza sociocultural y natural de su comunidad.

Desde nuestra perspectiva, un docente comprometido con tales principios puede valorar el potencial de las TIC y su posible uso para apoyar el desarrollo de ciertas capacidades en los estudiantes. De la actitud que asuma ante las TIC, su uso en el salón escolar y sus efectos en la formación de los niños y niñas, dependerá que estos se familiaricen con tales recursos, valoren su potencial, y los utilicen en beneficio propio y de la comunidad.

La experiencia empírica ha mostrado que para los niños, niñas y jóvenes, el computador no representa una amenaza, todo lo contrario; si son motivados a trabajar con dichos recursos, estas herramientas los animan y los seducen, precisamente por su carácter lúdico y por el poder de la imagen. La resistencia se ha observado más en los docentes. Lo preocupante es que si esto sigue ocurriendo, paradójicamente, la escuela, sin proponérselo, está contribuyendo al incremento de las desigualdades, en particular, a la reproducción de la brecha digital, lo cual es grave para las zonas rurales, por las limitadas oportunidades educativas formales y no formales que se tienen en estas regiones.

En el mejor de los casos, los niños, las niñas y los jóvenes logran acceder a la computadora y a Internet en otros espacios, lo cual no garantiza su mejor utilización. La intención es aprovechar el potencial que ofrecen las TIC para el desarrollo integral de los estudiantes y de las mismas comunidades.

Se trata de la construcción de un sentido del cambio social que le otorgue a las TIC una posición legítima, un sentido comprensible, en el cual se define a las personas y a las comunidades como actores y no solo como simples beneficiarios. 
Es un hecho que el uso de las TIC, al igual que otros recursos, tiene sus riesgos, pero también ofrecen oportunidades para encontrar respuestas a situaciones concretas. El reto es construir una práctica educativa participativa, que le permita a las poblaciones rurales comprender o entender la cultura del mundo globalizado, sin perder sus saberes y herencia cultural.

Cualquier uso que se dé a las TIC en el medio rural debe estar relacionado con una estrategia participativa y deliberada, cuya finalidad sea encontrar respuestas a las necesidades apremiantes que tienen las comunidades, pero, para ello, se requiere primero confirmar la satisfacción de sus necesidades básicas. Luego, inversión y conectividad. Además, la elaboración de contenidos y de propuestas pedagógicas que respondan a sus necesidades educativas, lo mismo que una práctica educativa comprometida de crítica y autocrítica, en función de estas poblaciones.

¿Qué no debemos hacer? Repetir el discurso oficial sobre el uso de las TIC y con ello promover una educación descontextualizada.

¿Debería ser una prioridad de las políticas estatales la conectividad en las zonas rurales? Desde nuestra perspectiva sí, es un derecho que tienen las poblaciones de estas zonas. Internet es una opción muy poderosa, si se utiliza de manera adecuada. El no hacerlo, se estaría, sin ser la intención, apoyando la brecha digital. Lo importante es no perder la perspectiva pues no hay atajos tecnológicos para el desarrollo humano. La sociedad, la "comunidad", la persona, con sus aspiraciones, temores, conflictos y decisiones, es el único motor real del futuro. Hacia eso, es lo que debemos canalizar nuestros esfuerzos.

Parafraseando a Oliveira (1976), se concluye afirmando que el papel de la educación rural en los países del tercer mundo es ejercitar la imaginación en busca de soluciones totalmente nuevas; jamás repetir las soluciones históricas del mundo desarrollado.

\section{REFERENCIAS}

Anfossi, A., Acuña, A. L. \& López, E. (2002). Ambientes de aprendizaje informatizados, construccionismo y currículo escolar. Consultado el 12, junio de 2006 en: http://lsm.dei.uc.pt/ ribie/docfiles/txt200372915425Ambientes\%20de\%20aprendizaje.pdf

Arredondo, M. A., Catalán, R., Montesinos, J., y Monsale, S. (2001). Aproximación etnográfica en la introducción de nuevas tecnologías de información y comunicación en dos escuelas rurales del centro sur de Chile. Consultado el 18, junio, 2006 de www.red-ler.org/introducciontics-educacion-rural-chile.pdf

Bossio, J., López, J., Saravia, M. \& Wolf, P. (2004). Desarrollo rural y tecnologías de información y comunicación. Experiencias en el Perú: lecciones aprendidas y recomendaciones. Recuperado 22, junio de 2006 de http://www.itdg.org.pe/archivos/tic/desarrolloytic.pdf

Fundación Omar Dengo. (2005). Informe Estadístico del PRONIE MEP-FOD I y II Ciclos al 03 de enero 2005. Departamento de Investigación, FOD. Recuperado el dia, mes de año de http:// www.fod.ac.cr/pronie/estadisticas/EstadisticasDis2004/COBERTURA\%20PRONIE\%20 I-II\%20ZONA\%20MEP\%20(03-01-05).xls 
Fundación Omar Dengo. (2006). Internet y aprendizaje en línea. Consultado el 26 de junio de 2006 de http://www.fod.ac.cr/contenidos/instituto/nexos/infoGeneral.htm.

Instituto Nacional de Estadística y Censos (2001). "IX Censo Nacional de Población y V de Vivienda: Resultados generales". Consultado el dia, mes de año de en http://www.inec.go.cr/ INEC_DIS/Publicaciones/archivos\%20SerieCensal\%20xls/ResulCenso2000.pdf

Marchesi, A. y Martín, E. (2003). Tecnología y aprendizaje. Investigación sobre el impacto del ordenador en el aula. Madrid: Instituto IDEA.

Ministerio de Educación Pública [MEP]. (2004a). Relanzamiento de la educación costarricense. Recuperado de http://www.mep.go.cr/Relanzamiento.asp

Ministerio de Educación Pública [MEP]. (2004b). Plan de Acción de la educación para todos 20032015. San José, Costa Rica.

Ministerio de Educación Pública [MEP]. (2005). I Informe de Seguimiento del Plan de Acción EPT. San José, Costa Rica.

Ministerio de Educación Pública [MEP]. (s.f.). Costa Rica. Marco Institucional y Contexto. San José, Costa Rica. Consultado el día, mes de año de catedradh.unesco.unam.mx/catedradh2007/ PresentacionExperiencias/webEducacionDemocracia/docbas/COSTA_RICA.pdf

Monge, R. y Hewitt, J. (2004). Tecnologías de la información y las comunicaciones (TICs) y el futuro desarrollo de Costa Rica: el desafío de la exclusión. San José, Costa Rica, Academia Centroamericana. Consultado el 22, junio de 2006 de http://www.colombiadigital.net/informacion/docs/TicFut_vg_d.pdf

Mora, R. y Ramos, P. (2002). Educación y conocimiento en Costa Rica: Desafíos para avanzar hacia una política de Estado (Aut), IX Informe Estgado de la Nación en Desarrollo Humano Sostenible (pp. 5-116). San José, Costa Rica: Programa Estado de la Nación.

Oliveira, L. (1976). Mutaciones en la educación. Argentina: Editorial Humanitas.

Programa Estado de la Nación en Desarrollo Humano Sostenible. (2005a). Informe Estado de la Educación 1. San José, Costa Rica: Programa Estado de la Nación, Consejo Nacional de Rectores.

Programa Estado de la Nación en Desarrollo Humano Sostenible. (2005b). XI Informe Estado de la Nación en Desarrollo Humano Sostenible. San José, Costa Rica: Programa Estado de la Nación.

Programa Estado de la Nación en Desarrollo Humano Sostenible. (2006). XII Informe Estado de la Nación en desarrollo humano sostenible. San José, Costa Rica: Programa Estado de la Nación. 
Tedesco, J. C. (2003). Las Tics y la desigualdad educativa en América Latina. Consultado el día, mes de año de http://www.enlaces.cl/ocde/doc/Conferencias/TICs_Desigualdad(3).doc

UNESCO. (1996). La Educación encierra un tesoro. Informe a la UNESCO de la Comisión Internacional sobre la Educación para el siglo XXI. Madrid: Santillana, Ediciones UNESCO. 